\title{
A Taxonomy Survey on Energy-aware Virtualized Data Cenetrs Algorithms and Techniques
}

\author{
Sanjay Patel \\ Department of Computer Engineering, LDRP-ITR, Gandhinagar, Gujarat, India \\ Email: sanjaypatel54@gmail.com
}

\begin{abstract}
Cloud computing is a novel paradigm which defines a new style of deploying systems. It realizes the vision of delivering Computing as a Utility. The demand for Cloud infrastructure has expanded with rapid growth of computing applications and data thereby amplifying energy-consumption. These results in heightened cost of ownership, reduced return on investment, narrowed profit margin, decreased reliability and availability of datacenter resources, and above all it adversely affects the environment by increases carbon footprint. In this context energy management system has been introducing, also it gives battery feeds mobile devices which improves the life of battery lifetime energy consumption has to be used. For data centers and servers these all techniques are used for improving energy consumption for that the system required specific techniques and methods. In this whole paper, we discuss about the available techniques and systems for various hardware systems, servers, virtualization, operating systems, and virtualization data centers levels. The objective of these techniques is given brief view of recent or latest research in saving of energy sector and identifies the common parameters for improving energy efficiency. This paper also discusses about a common approach for energy efficient algorithms and techniques.
\end{abstract}

Keyword - Cloud computing, Green Computing, Green IT, VM consolidation, VM Placement, VM Selection, Host Under load Detection, Host Overload Detection

\section{INTRODUCTION}

For the system performance, energy and power consumption both defined as certain terms of total system task performed by the hardware resource. Power is nothing but an overall power rate is perform by the systems and Energy is the over the period of time total amount work performed by the systems which shown in figure 1. We consider power and energy in these systems, and power and energy measured in given units. It is measured by watt hour and watts. One watt considers the work done by using Ampere is the difference by the single which is transferred through. A kWh consider as one thousand watts running for the single hour and total no of power and energy equivalent. Energy Consumption and power consumption factors defined by in below equation for energy aware sector [7].

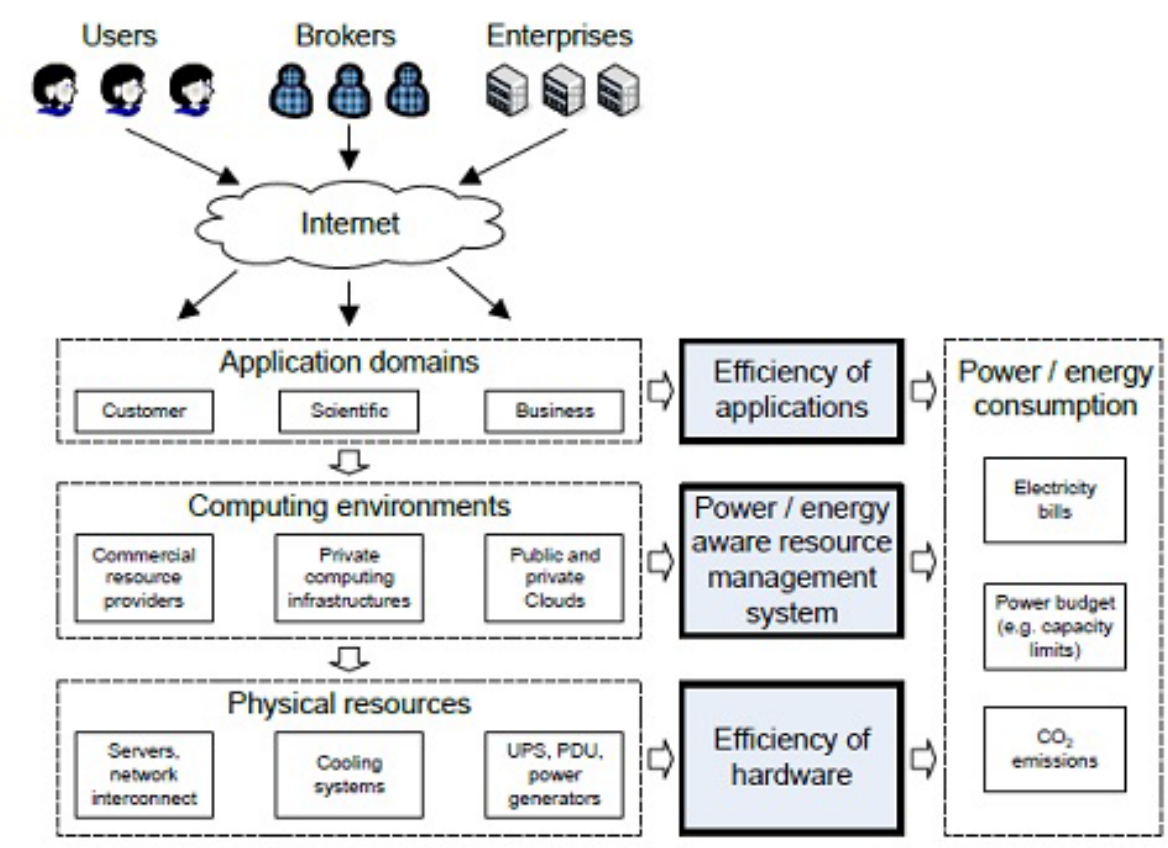

Figure 1. Energy Consumption at Computing Systems [6] 
1. POWER AND ENERGY MODEL

$$
\begin{aligned}
& \mathrm{P} 1=\mathrm{Ws}=\mathrm{T} 1 \\
& \mathrm{E}=\mathrm{P} 1 \mathrm{~T} 1
\end{aligned}
$$

Where $\mathrm{P} 1$ is power, $\mathrm{T} 1$ is a total time period, Ws is the work performed by the systems in that $\mathrm{T} 1$, and the author considers $\mathrm{E}$ is energy. Power and Energy different play a crucial role and it is very important for the system because reduce the energy every time power consumption cannot be used. For one standard example lowering CPU performance, the power consumption can be decreased. In this particular case, a program may take a longer time to complete its execution where same energy consumption can be used. The other issue the reduction of the power consumption will be the decrease the cost of all available infrastructure, the cost is related to various infrastructure equipment like PDU, power distribution elements, power generators device and various cooling systems. Using this all energy saving techniques energy consumption will decrease and it is directly affected by reduction of the all over energy bills [7].

\section{STATIC AND DYNAMIC POWER CONSUMPTION}

For generating or using static and dynamic power the CMOS circuits complementary required.

- Static power consumption independently the current or power leakage in any active or passive circuit. This static power also determined by transistors and various processors technology. For static power reduce required to improve system design which is low level and architecture. Using the circuit activity power consumption dynamically created, it also de- pends on the various

- Usage scenario, I/O activity, and various clock rates. Switched Capacitance and various short circuit current are both the various sources of the power consumption. The main power consumption by the short circuit current up to 10 to $15 \%$, without compromising the system performance yet no way found to reduce this power consumption value.

For dynamic power consumption, the primary source is switched capacitance, that's why the dynamic power consumption defined for the system as in

$$
\text { Pdy }=\mathrm{a} 1 \mathrm{c} 1 \mathrm{v} 12 \mathrm{f} 1
$$

Where a1 call the switching activity, C1 defined as a physical capacitance for the system, V1 call as a supply voltage for the system, and f1 defined for various clock generation frequency. Using low-level system design the various values of capacitance and switching activity are determined [7].

\section{SOURCES OF POWER CONSUMPTION}

As per Intel Research Labs report physical server is a main part of power consumed which includes various Processors, RAM and losses due to the power supply inefficiency in Figure 2. As per data show in a figure 2, the Processors no longer dominates power consumption by a physical server. That's why techniques required continuous improvement of the processor power efficiency and application of power saving techniques that enable active low-power modes [8].

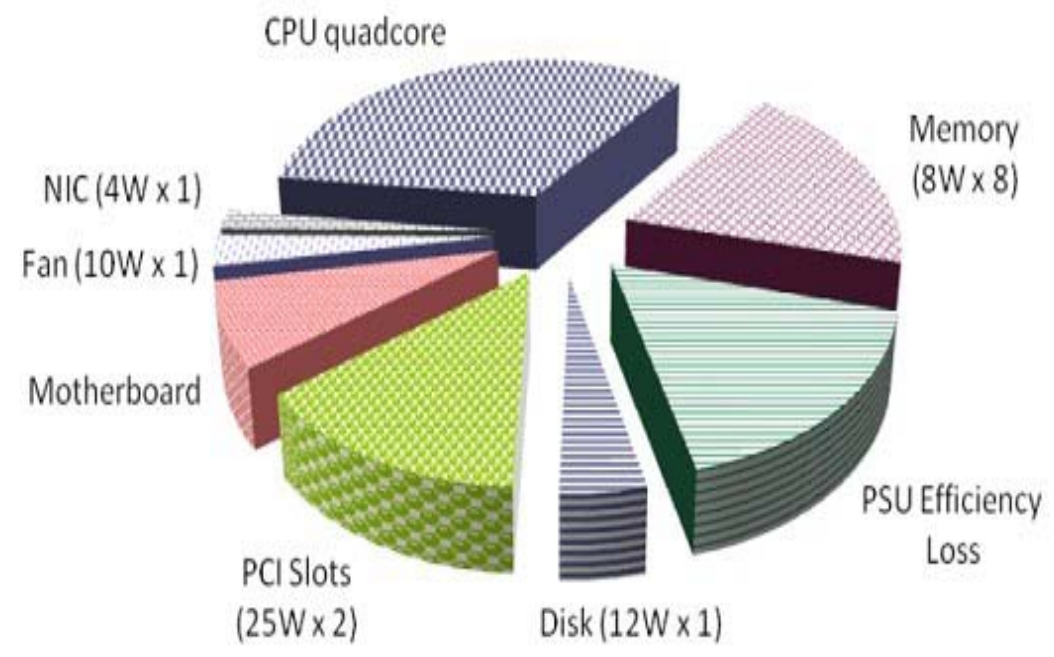

Figure 2. Sources of Power Consumption [3]

As per some report, multi-core processors are much more efficient than conventional architectures. There is another reason to reduce the power consumed by CPU relatively to the whole system is an adoption of multi-core architectures. The problem of VM consolidation can be subdivided into four sub-problems [4]. These are shown in the Figure 2 have presented Dynamic consolidation with migration control [9]. We will limit our 
discussion to VM placement strategies. [9] Have presented Dynamic consolidation with migration control in Figure 4. Their aim is to ensure more stability for VMs with steady demands and that VMs with steady demand do not migrate. They have contributed an LP formulation and heuristics to control VM migration, which prioritizes VMs with steady capacity [9].

\section{NEED FOR ENERGY EFFICIENCY}

Computing applications and data are growing so quickly that increasingly larger servers and disks are needed to process them fast enough within the required time period. Data centers are expensive to maintain and unfriendly to the environment. Over $90 \%$ of a data center's electricity is consumed by the information technology equipment (namely server, storage, and network) and cooling facility, with the rest dedicated to lights and everything else. Massive amounts of electricity needed to power and cool numerous servers hosted in the data centers. The providers need to ensure that profit margin is not dramatically reduced due to high energy costs. The need for energy-efficient cloud computing arises due to [10]:

- Increased cost of ownership

- Reduced Return on Investment

- Increase in carbon footprint

- Decreased reliability and availability of data center resources

- Pressure from Govt. to reduce carbon footprint

In this figure 3 data center various energy consumption explain. Three categories of data center are display. First one is server, which is consume $50 \%$ energy consumption. Second one is storage which consume $35 \%$ energy consumption and network devices consume the 15\% of overall energy consumption. This figure 3 also gives the detail explanation about the layer and how much energy consume by every layer [11].

\section{GREEN COMPUTING}

Green computing generally refers as energy saving while computing. The terms Green IT, ICT and Green Commuting is the study of or we can say practice environmentally IT or sustainable computing.

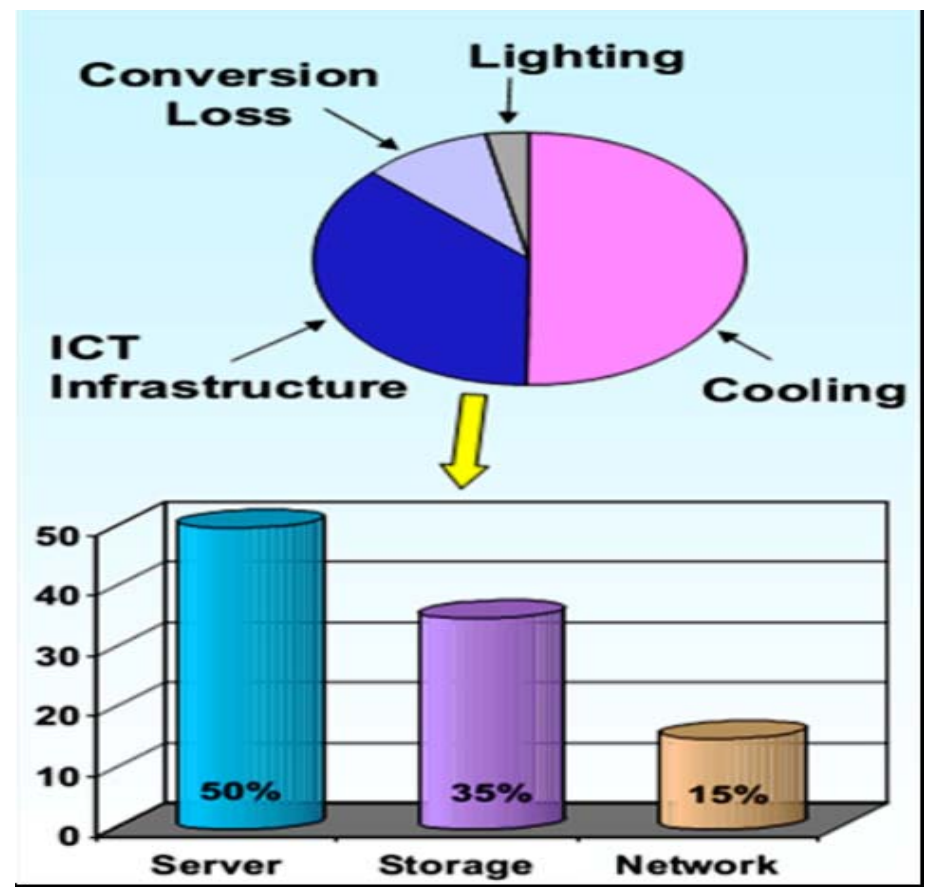

Figure 3. Data center Energy Consumption [7]

Some author notes this can include (San Murugesan) "designing, manufacturing, using, and disposing of computers, servers, and associated subsystems- such as monitors, printers, storage devices, and networking and communications systems - efficiently and effectively with minimal or no impact on the environment” [12]. 


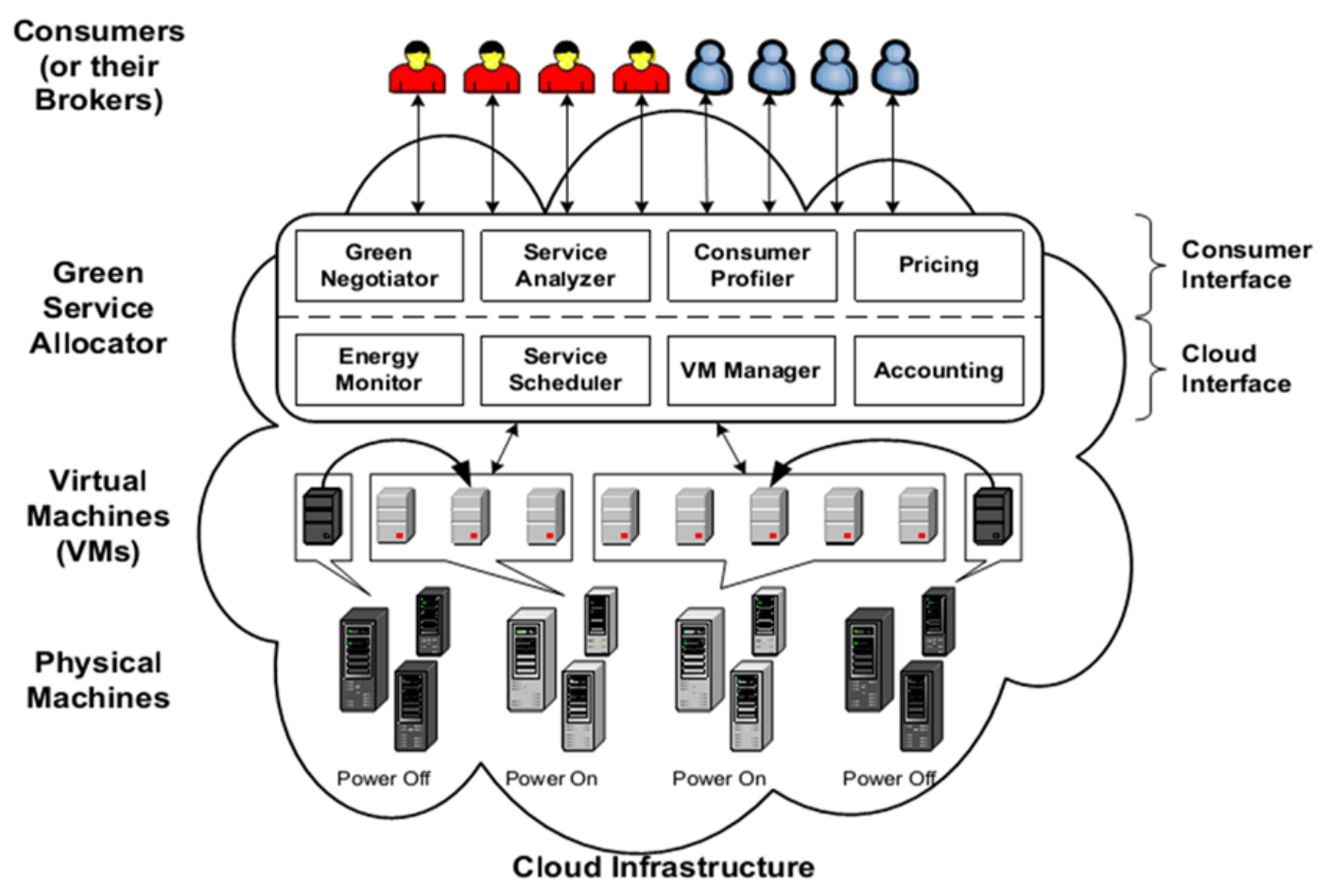

Figure 4. Green Cloud Architecture [12]

In this figure the architecture supporting energy saving service allocation in Green cloud computing infrastructure. This architecture basically divided into four parts, it means that various four entity play a most important role:

1) Brokers/Clients: Cloud users, various consumers or brokers require to access service request or they can submit various services request to the cloud anywhere from the world. It is very important thing for cloud providers to differentiate between cloud consumers and various users or clients of various deployed service. According to various numbers of users accessing the cloud services, the consumers which present the workload whether it is deploying web application or any simple application.

2) Allocator for Green Resource: It is provide the interface between brokers/clients/consume and cloud infrastructure. To support the energy efficient resource management the various components acts as the most important role from green resource allocator:

- Green Negotiator: As per clients or users Quality of Service (QoS) requirements and various energy saving scheme, it's negotiates with the various users or various brokers or Consumers to finalize the Service level agreements penalties and prices (when QoS parameter SLA violation or broken). When considering case of web-based application QoS metric serve 95\% request within or less than three seconds.

- Service Analyzer: Whether accepting or reject request it's analyze and combine the various requirements of services which request are submitted by users. It's required from energy monitor and VMM layer the information of latest load energy information.

- Consumer Profiler: Based on requirements of consumers or clients service provider granted or allow to access priority basis compare to other consumers or clients.

- Pricing: The main aim of the service provider is to provide effective services and economy better services to the consumers or clients and also decide how service request are changed.

- Energy Monitor: Energy monitor observe all the available physical machine and decide that which PM should be on or off based on service accessing conditions.

- Service Scheduler: Based on users requests assign the virtual machine to the specific service and decide that the when and which VMs added based on demanding various services.

- VM Manager: The main objective of VM manager is to track the record or details of each and every VMs and it is responsible for the charge various VMs migrating across physical machines.

- Accounting: In this accounting section it maintains the resource usage by the various consumers and counts the cost according to the usage, also it keeps the record for improving the service allocations requirements and decisions. 
- Virtual Machines: There are various requests come from consumers and brokers for accessing the services, based on service requests all available VMs started and stopped on various physical machines for providing flexibility and scalability to configure various physical machines VMs. Based on various physical machine operating systems various VMs can also run various applications concurrently. In order to save energy various algorithm and techniques (using DVFS) may use, also some ideal resources put in a category of low power state. VMs across physical machines dynamically migrate and workload also is consolidated.

- Physical Machines: To meet the service demands from the consumers it provides the physical infrastructure to create various visualized resources or virtual machines.

\section{POWER MANAGEMENT TECHNIQUES}

Power management terms are generally similar as energy management both provide power saving or management techniques in data centers. According to the figure, power management techniques can be divided into two categories referred as static and dynamic. SPM includes various available methods which are optimized that can apply at the same time of architecture level design, system, architecture and logical levels. Generally, circuit optimizations basically focus on reduction of the switching activity power from various logic gates from circuits. It also refers to transistor level complex gate design and sizing. Dynamic Power Management techniques generally refer as according to systems current resource requirements as it provides the methods and strategies for runtime adaption [13].

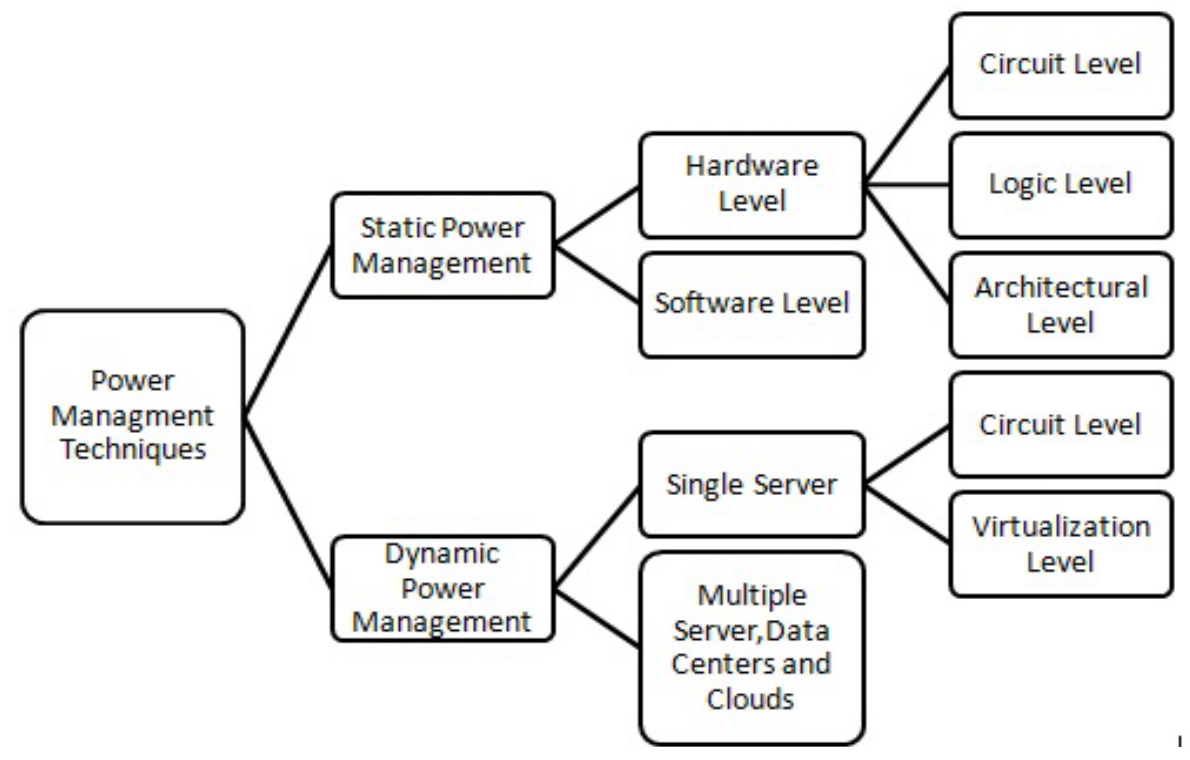

Figure 5. Power Management Techniques [13]

According to the current performance requirements, it is allowing the dynamic power states adjustments based on systems experience variable load during the requests operations time. It also provides various methods to the VMs. This is the major assumption enabling DPM.

Also DPM provides the assumption related to the workload. According to the second assumptions, workload can be predicated. As per figure 5, DPM techniques divide by various levels at which DPM applied. One is Hardware and another is software.

DPM the first type Hardware DPM it's include various hardware components, but it is classified by Dynamic Performance Scaling (DPS), such as DVFS, and complete or partial Dynamic Components Deactivation (DCD) during inactivity periods. In other side Software, DPM methods are used for utilizing system performance as per their various policies apply by dynamic power management techniques. The various problems of management of power have been various context-specific management, also its Operating System level management of various servers like virtualized and non-virtualized, is defined by multiple node systems, various clouds, data centers and homogeneous and heterogeneous clusters. For CPU enabling low power active modes DVFS play a most important role to create broad dynamic power range. Also, these techniques are used for coordinating multiple servers. Sometimes to narrow dynamic power range to consolidate power range of services to limited numbers of servers to switch on and off or put in sleep mode [13]. 
The quality of Service is one of the important characteristics of any cloud computing architecture. The QoS must be reliable for all applications. One of the important factors is Service level agreement (SLA) provided by QoS and its characteristics are minimal throughput, latency must be maximal or response time must be higher for the proposed systems. Between VMs sharing similar computing node modern or latest virtualization architecture or technologies ensure the performance isolation. The problem is some VMs may not get a required number of resources due to variability workload and aggressive consolidation. In some of the worst cases performance losses in various terms like timeouts, failures or increased response time. The main requirements for users is QoS, while QoS provide to the users the cloud providers must follow power-performance trade-offs and minimization of the power consumption.

\section{VM CONSOLIDATION}

VM consolidation can be defined as the process of gathering several virtual machines (VMs) into a single physical server. This is made possible by the concept of virtualization, which is the foundation technology of cloud computing. Between OS and Hardware, an abstraction introduces by virtualization. Single or multiple physical machines are the divide into a number of parts or slices are called Virtual Machines (VMs). As per dedicated view of physical resource each and every virtual machine can accommodate and it is ensuring the failure recovery and performance ensuring. This technique can allow creating no of the virtual machine from all available hardware; and advantage for the improvement the performance and utilization of resources. This layer is generally used for the interface between hardware and os, and virtual machine monitor responsible for this. The main role of VMM layer manages the resource allocation and multiplexing of the resources. There are basically two ways using this Virtual Machine Monitor can participate in energy saving and power management [15]:

Below is that the planned formula to calculate the ability consumption of the data center by the various architecture like heterogeneous work VMs consolidation. The formula using given host list for input parameters purpose and provides migration list of power consumed by the data center and various list of VMs. The very first step of algorithm identify the no of VM decreasing order for hardware utilization and so it process repeat appearance for the simplest which from a host which VM to migrate and additionally account the data center power consumption. The basically objective of algorithm give the migration list and power consumption by the data center [6].

- A VMM play a monitor the system and power aware os performance and DVFS and some another DCD techniques to the system or hardware components.

- Based on application level knowledge a VMM layer provide some power and energy management policies by the guest operating systems, and various operating systems mapping power management commands given of various available VMs power state changes in hardware layer, it's also power limits system to enforce coordinated manner.

Cloud computing world there is three most important virtualization technology solutions are: KVM, Xen hypervisor, and VMware solutions. These all systems are supported by power and energy management. These all are the virtualization benefits [15]:

- Fault tolerance improve

- Same compute node performance isolation between Applications (The VMs view as system dedicated node or physical resource for the various consumers)

- One host and another physical host using live migration the ability easily move VMs(In data center level for energy efficient dynamic VM consolidation the ability to move VMs migrate)

- Support for hardware and software heterogeneity.

Benefits provided by the VM consolidation are:

- Increases resource utilization

- Reduces electric power consumption cost

- Important in case of unpredictable workload (as per users demands all VMs can resize and online or offline migrated to the physical resources) 


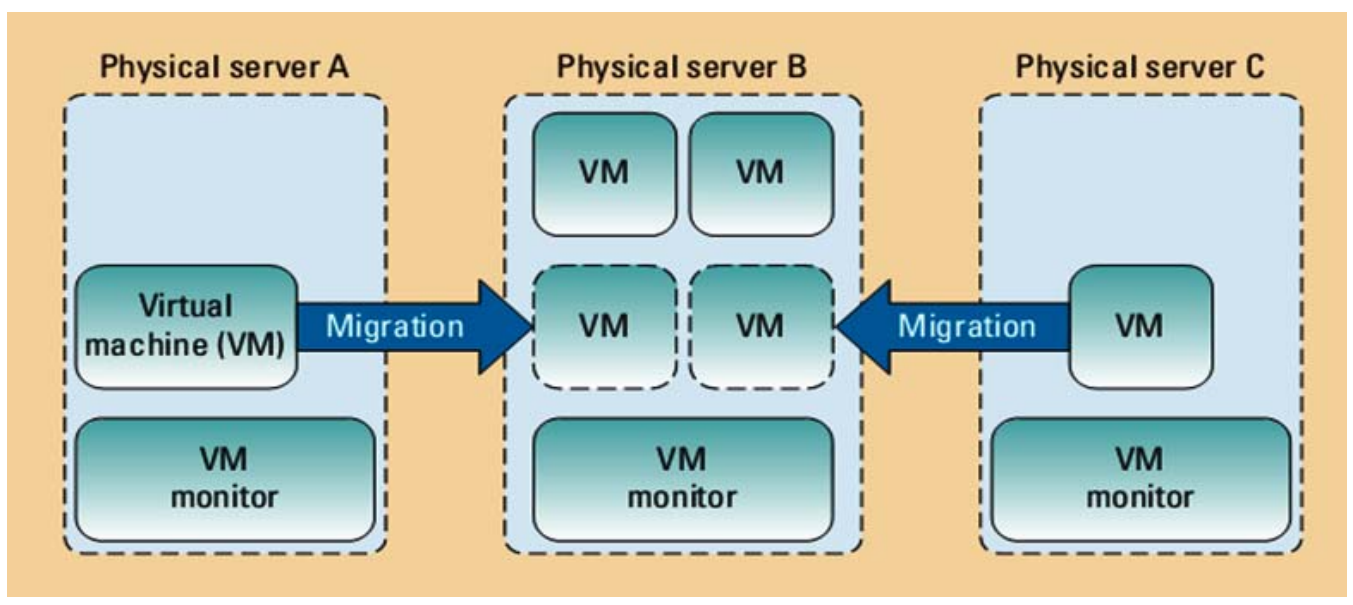

Figure 6. VM Consolidation [15]

\section{A - VM CONSOLIDATION ALGORITHMS}

VM consolidation algorithms can be devised based on the following characteristics [16]:

1. Virtualization: The approach virtualization resource of the data center. This is the important characteristics considering the proliferation of Cloud data centers.

2. System resources: The resource utilization of single physical resource the types are various system resource or hardware resource CPU which is multiple the system takes into account.

3. Target systems: The two types of system architecture defines whether it's a heterogeneous or homogeneous. In an example of large-scale data centers the heterogeneity used for efficient handling, as the capacity of systems incrementally increased over a time resulting in heterogeneous resources of the systems.

4. Goal: The main aims of the systems is to decrease energy consumption or power consumption the various challenges are used and meeting with appropriate power pay or account.

5. Power Saving Techniques: A DVFS algorithm used apply DPS for resource uses; in whole servers DCD including power states of switching; or to minimize energy and power consumption the workload or VM consolidation can be used.

6. Workload: To ability to handle the random workload and the system is application specific or it focuses on specific application category, this includes HPC and various services.

7. Architecture: To fulfill requirements of resource management to be centralized the algorithm must run as distributed or master node.

\section{FUTURE RESEARCH DIRECTIONS}

Based on literature review the following are future research directions [17] [18].

- Advanced Distributed VM Placement Algorithms: By using several requests in buffering it is decide buffer size and consider the request using VM placement algorithm.

- VM Network Topologies: Using design and develop of various network devices and cost for transferring data provide efficient reallocation.

- Exploiting VM Resource Patterns: To search patterns in order to cloudlets, explore workload and more resource provisioning

- Thermal-Aware Dynamic VM Consolidation: To provide cooling take care about operating cost and reallocate VM accordingly

- Dynamic and Heterogeneous SLAs: Based on the SLA satisfaction resources can be allocated to the clients.

- Competitive Analysis of Dynamic VM Consolidation Algorithms: To increase performance guarantees VM selection algorithm and VM underload detection algorithm introduce 


\section{ENERGY EFFICIENT VM CONSOLIDATION CHALLENGES}

There are various challenges of energy-efficient VM consolidation under the various QoS requirements, In particular below mention the research problems [19] [20]:

- QoS requirements based on workload: From the QoS Matrices derived workload independent and find out systems performance

- Migration when required for VMs: There are two basics processes for dynamic VM consolidation:

o Migration is required when performance degradation occur; and

o When under loaded VM detected migration is required to improve utilization

Based on various QoS constraints with minimize energy consumption which best VM required to Migrate from physical server [20].

- VMs to migrate: Based on the request which VM migrate from which server

- VMs Migration Location: Based on the required VM to migrate to satisfy the performance

- Switch on/off node: Based on dynamic switching and VM consolidation eliminating the data center power consumption

- Design dynamic VM consolidation algorithms: To improve QoS paraments like scalability and reliability it is required to design dynamic consolidation algorithms for cloud data centers

\section{CONCLUSIONS AND FUTURE DIRECTIONS}

From the literature survey it is concluded that, from all above techniques and architecture efficient power management required. For reduction of energy consumption it plays an important role. There are number of challenges in the field of cloud computing. Numbers of users have been increasing day by day. According to the all user's requirements and to fulfill all users' services demands there is a need of large number of data centers. When we are increasing large number of data centers, the energy consumption is very high. As per McKinsey report" the energy cost in data centers double every five years and data centers energy bill is \$ 11.5 billion in year 2010”. And Google reports that the energy cost in data centers per year is double every two years. The motivation behind this research is the need to develop more energy efficient algorithm for data centers and this data centers fulfill below requirements. The need of energy cost is double every five years according to McKinsey report. The energy cost of data centers for

Every five years is $\$ 11.5$ billion in year 2010. To reduce the energy consumption, it is required to develop energy efficient algorithm and identify the parameters for better development of algorithm. For migration strategies it is required to develop VM migration algorithm to decrease the migration. When migration is decrease the energy cost is automatically decrease. That's why some optimal algorithm is required for minimal migration. The main aim of cloud data centers is to provide services to the users with minimal cost. When we are talking about services, the services must be Quality of Services both for user and cloud providers side. The main issues of today scenario is $\mathrm{CO} 2$ emission, using VM consolidation algorithm try to minimize environment $\mathrm{CO} 2$ emission. For these reasons, it is required to design and develop VM consolidation and VM migration algorithms.

\section{REFERENCES}

[1] Si-Yuan Jing, Shahzad Ali, Kun She,Yi Zhong, State-of-the-art research study for green cloud computing, The journal of supercomputing, Springer, Volume 8, July 2013.

[2] P.Mell, and T. Grance, the NIST definition of cloud computing, special publications, 2013.

[3] Eugen Feller, Louis Rilling, Christine Morin, Energy-Aware Ant Colony Based Workload Placement in Clouds, INSTITUT NATIONAL DE RECHERCHE EN INFORMATIQUE ET EN AUTOMATIQUE, ISSN 0249-6399, inria-00594992,Volume 1, May 2011.

[4] Rajkumar Buyya, Anton Beloglazov and Jemal Abawajy, Energy-Efficient Management of Data Center Resources for Cloud Computing: A Vision, Architectural Elements and Open Challenges, In proceedings of the international conference on parallel and distributed processiong techniques and applications (PDPTA), 2010.

[5] Anton Beloglazov, Rajkumar Buyya, Energy Efficient Resource Management in Virtualized Cloud Data Centers, 10th IEEE/ACM International Conference on Cluster, Cloud and Grid Computing, 2010.

[6] Anton Beloglazov, Rajkumar Buyya, Young Choon Lee, and Albert Zomaya, A Taxonomy and Survey of Energy-Efficient Data Centers and Cloud Computing Systems, 2010.

[7] L. Minas and B. Ellison, Energy Efficiency for Information Technology: How to Reduce Power Consumption in Servers and Data Centers. Intel Press, Aug. 2009.

[8] L. A. Barroso and U. Holzle, The case for energy-proportional computing, Computer, pp. 33.37, 2007.

[9] G. Wei, J. Liu, J. Xu, G. Lu, K. Yu, and K. Tian, The on-going evolutions of power management in Xen,. Intel Corporation, Tech. Rep., 2009.

[10] VMware Inc., vSphere resource management guide, 2009.

[11] How VMware virtualization right-sizes IT infrastructure to reduce Power consumption, 2009.

[12] Qumranet Inc, KVM: kernel-based virtualization driver, White Paper, 2006.

[13] R. Nathuji and K. Schwan, Virtualpower: Coordinated power management in virtualized enterprise systems, ACM SIGOPS Operating Systems Review, volume 41, no. 6, pp. 265.278, 2007. 
[14] R. Raghavendra, P. Ranganathan, V. Talwar, Z. Wang, and X. Zhu, No "power” struggles: Coordinated multi-level power management for the data center,. SIGARCH Computer Architecture News, Volume 36, no. 1, pp. 48.59, 2008.

[15] M. Stillwell, D. Schanzenbach, F. Vivien, and H. Casanova, Resource allocation using virtual clusters, in Proceedings of the 9th IEEE/ACM International Symposium on Cluster Computing and the Grid (CCGrid), Shanghai, China, pp. 260.267, 2009.

[16] A. Verma, P. Ahuja, and A. Neogi, pMapper: power and migration cost aware application placement in virtualized systems, in Proceedings of the 9th ACM/IFIP/USENIX International Conference on Middleware. Springer-Verlag New York, Inc., pp. 243.264, 2008.

[17] R. Buyya, A. Beloglazov, and J. Abawajy, Energy-Efficient management of data center resources for cloud computing: A vision, architectural elements, and open challenges, in Proceedings of the 2010 International Conference on Parallel and Distributed Processing Techniques and Applications (PDPTA), Las Vegas, USA, July 12-15, 2010.

[18] K. H. Kim, A. Beloglazov, and R. Buyya, Power-aware provisioning of cloud resources for real-time services, in Proceedings of the 7th International Workshop on Middleware for Grids, Clouds and e-Science (MGC), Urbana Champaign, Illinois, USA, pp. 1.6, 2009.

[19] Dzmitry Kliazovich, Pascal Bouvry, Samee Ullah Khan, GreenCloud: a packet-level simulator of energy-aware cloud computing data centers, Springer Science+Business Media, LLC 2010.

[20] Anton Beloglazov, Rajkumar Buyya, Energy Efficient Resource Management in Virtualized Cloud Data Centers, 11th IEEE/ACM International Conference on Cluster, Cloud and Grid Computing, 2011.

[21] Lizhe Wang, Gregor von Laszewski, Fang Huang, Jai Dayal, Tom Frulani, Geoffrey Fox, Task scheduling with ANN-based temperature prediction in a data center: a simulation-based study, Springer-Verlag London Limited 2011.

[22] Anton Beloglazov, Jemal Abawajyb, Rajkumar Buyyaa, Energy aware resource allocation heuristics for efficient management of data centers for Cloud computing, Future Generation Computer Systems, pp 755.768, 2012.

[23] Chia-Ming Wu, Ruay-Shiung Chang, Hsin-Yu Chan, A green energy efficient scheduling algorithm using the DVFS technique for cloud datacenters, Elsevier Future Generation Computer Systems, 2013.

\section{AUTHOR PROFILE}

Sanjay Patel working as an Assistant Professor at LDRP-ITR. I completed Ph.D. in Computer Engineering from CSPIT, Charotar University of Science and Technology, Changa, India in 2017. I received Master degree in Computer Science and Engineering from Nirma Institute of Technology, Nirma University, Ahmedabad. My research interest is Cloud Computing, Internet of Things and Distributed Computing. I am interested in research work that has great impact on society and nation developments. 